\title{
DELAYED-ONSET HEPATIC ENCEPHALOPATHY INDUCED BY ZOLPIDEM: A CASE REPORT
}

\author{
doi: 10.1590/S1807-59322008000400028
}

Vitor Carlos Santos da Silva, Paulo Lisboa Bittencourt, Sylvania Pinho, Andréa Ribeiro Cavalcanti, Cláudio Celestino Zollinger

\section{INTRODUCTION}

Hepatic encephalopathy (HE) is a complex neuropsychiatric disorder associated with either hepatocellular insufficiency and/or the presence of porto-systemic shunts. It is thought to be induced by altered neurotransmission mediated by enhanced gamma-aminobutyric acid (GABA)-induced inhibitory tone. ${ }^{1-3}$ The use of benzodiazepines are generally avoided in patients with advanced chronic liver disease (CLD) because they enhance GABA-mediated neurotransmission. Zolpidem is a non-benzodiazepine hypnotic that selectively binds to the omega-1 GABA-benzodiazepine receptor complex. Although it theoretically has the same propensity to induce HE, its shorter half-life when compared to commonly prescribed benzodiazepines like diazepam, alprazolam and clonazepan, makes it a reasonable choice for subjects with CLD. $^{4}$

We report a patient with CLD with a rapidly induced grade IV HE attributed to zolpidem that was promptly reversed by flumazenil.

\section{CASE REPORT}

A 73-year-old female with a past history of Lewy body dementia, transient insomnia and arterial hypertension was admitted to our unit due to jaundice and ascitis dated one week before hospitalization. She was taking quetiapine $12 \mathrm{mg} /$ day and rivastigmine $12 \mathrm{mg} /$ day for approximately six months. Physical examination disclosed involuntary movements of the upper limbs, temporal and spatial disorientation, jaundice and moderate abdominal and ankle swelling.

Unidade de Gastroenterologia e Hepatologia do Hospital Português - Salvador/BA, Brasil.

Email: plbbr@uol.com.br
Laboratory data revealed AST 53 IU/L, ALT 65 IU/L; alkaline phosphatase 187 IU/L; gammaglutamyltranspeptidase $117 \mathrm{IU} / \mathrm{L}$; albumin $1.3 \mathrm{~g} / \mathrm{dL}$; bilirubin 3.48 $\mathrm{mg} / \mathrm{dL}$ and INR 1.75 .

Screening for hepatitis B surface antigen (Enzyme Linked Immuno Sorbent Assay - ELISA, Abbott Laboratories, North Chicago, IL) and anti-hepatitis C virus (anti-HCV) antibodies (ELISA II, Ortho Diagnostic System, Raritan, New Jersey) yielded negative results, as did anti-smooth muscle, antinuclear, anti-liver kidney microsome type 1, anti-liver cytosol type 1 and antimitochondrial antibodies. Other causes of CLD, such as hemochromatosis, alcoholic liver disease and alpha-1 antitrypsin deficiency, were ruled out by history and the appropriate laboratory tests. Ultrasound examination revealed a shrunken liver with irregular surface, no biliary dilatation and a $3-\mathrm{cm}$ hypoechoic nodule in the left lobe. Alpha-fetoprotein levels were $440 \mathrm{IU} / \mathrm{L}$. Esophagogastroduodenoscopy showed small esophageal varices and moderate hypertensive gastropathy.

In light of the diagnosis of cryptogenic cirrhosis complicated by hepatocellular carcinoma and ascitis, diuretics (spirolactone $100 \mathrm{mg} /$ day and furosemide $40 \mathrm{mg}$ / day) were started. Eight days after admission, the patient developed clinical and laboratory signs of sepsis and acute deterioration of consciousness characterized by torpor that evolved to a Glasgow coma scale of 5.

She was transferred to the intensive care unit (ICU). Before tracheal intubation and empiric antibiotic coverage, the use of intravenous $1 \mathrm{mg}$ flumazenil was attempted because the patient had received $10 \mathrm{mg}$ zolpidem only 12 hours before admission. Unexpectedly, the patient recovered to her basal state of consciousness 30 seconds after the bolus injection. After 14 days, the patient was discharged from the hospital, but unfortunately returned one month later with 
hepatic insufficiency. She received supportive measures and died one week later in the nursing faculty.

\section{DISCUSSION}

Zolpidem tartrate is a non-benzodiazepine imidazopiridine hypnotic with fast onset of action used to treat transient insomnia in doses averaging 5 to $10 \mathrm{mg}$ per day. In contrast to benzodiazepines, which bind nonselectively to all three omega gamma-aminobutyric acidbenzodiazepine (GABA-benzodiazepine) receptor subtypes, zolpidem binds selectively to the omega- 1 receptor, which could explain its lack of anxiolytic, muscle relaxant and anticonvulsant effects in its average therapeutic range. ${ }^{4}$ Due to this mechanism, it is not unusually prescribed to patients with CLD. Its half-life is short in normal subjects but is significantly altered in old patients and subjects with CLD due to hepatic metabolism. These characteristics could explain the rapid onset of HE in this aged patient with advanced CLD, as well as her prompt recovery with the use of flumazenil, a benzodiazepines and zolpidem antagonist. ${ }^{4,5}$
Sepsis and hypovolemia due to the use of diuretics could also be viewed as precipitants of $\mathrm{HE}$ in this patient, but the prompt recovery from HE with the use of flumazenil before fluid resuscitation and antibiotics argue against a major role for these triggers. It is worth mentioning that flumazenil has also been employed to treat HE unrelated to its hypnotic usage, but its role in this setting has not been validated in randomized controlled trials. ${ }^{6}$ However, it is more probable to assume that this patient's immediate recovery was due to a flumazenil-induced antagonism of the central nervous system effects caused by zolpidem because the patient had taken zolpidem only 12 hours before admission to the ICU due to HE.

In summary, HE could be precipitated by zolpidem in a similar manner to benzodiazepine hypnotics. Caution should be taken with the use of this drug in patients with advanced CLD because its half life could be severely prolonged with liver failure. It is notable that flumazenil is also an antagonist of non-benzodiazepine hypnotics, such as zolpidem, and could be efficaciously used to reverse HE induced by this class of drugs.

\section{REFERENCES}

1. Mas A. Hepatic encephalopathy: from pathophysiology to treatment. Digestion. 2006;73 Suppl 1:86-93.

2. Stewart CA, Cerhan J. Hepatic encephalopathy: a dynamic or static condition. Metab Brain Dis. 2005;20:193-204.

3. Shawcross DL, Damink SW, Butterwoth RF, Jalan R. Ammonia and hepatic encephalopathy: the more things change, the more they remain the same. Metabolic Brain Dis. 2005;20:169-79.
4. Swainston Harrison T, Keating GM. Zolpidem: a review of its use in the management of insomnia. CNS Drugs. 2005; 19:65-89.

5. Lheureux P, Debailleul G, De Witte O, Askenasil R. Zolpidem intoxication mimicking narcotic overdose: response to flumazenil. Hum Exp Toxicol. 1990;9:105-7.

6. Als-Nielsen B, Gluud LL, Gluud C. Benzodiazepine receptor antagonists for hepatic encephalopathy. Cochrane Database Syst Rev. 2004;(2):CD002798. 